\title{
Akutes Abdomen Erstversorgung
}

Ines Gockel • Martin Schröder • Hauke Lang • Carl C. Schimanski

\begin{abstract}
Abdominelle Schmerzen gehören zu den häufigsten Einweisungsdiagnosen in der Notaufnahme. Ob harmlos oder ernsthaft: Die Symptome ganz unterschiedlicher Erkrankungen sind oft ähnlich und unspezifisch. Trotzdem muss der Arzt schnell entscheiden: Droht Lebensgefahr? Muss man operieren? Dieser Beitrag definiert das akute Abdomen und erläutert die wichtigsten Strategien der Erstversorgung. Besonders relevant ist dabei eine interdisziplinäre Zusammenarbeit.
\end{abstract}

\section{Aspekte der Erstversorgung}

\section{Zeitnot}

Definition akutes Abdomen Das akute Abdomen ist eine akute Manifestation von Erkrankungen im Bauchraum, die schnell diagnostiziert und therapiert werden müssen. Seltener sind die Ursachen auch extraabdominal oder systemisch.

- Es handelt sich um eine vorläufige Bezeichnung für eine abdominelle Erkrankung, die oftmals zunächst nicht exakt differenzierbar ist.

Die häufigsten Leitsymptome sind

- Schmerz,

- Abwehrspannung,

- Übelkeit,

- eingeschränkter Allgemeinzustand und

- evtl. Störung der Kreislaufregulation

- bis zum Schock.

Schwierig für den primär behandelnden Arzt: Das Zeitfenster für die Erstversorgung ist limitiert. Außerdem erfordert das akute Abdomen eine interdisziplinäre differenzialdiagnostische Abklärung und Therapieentscheidung.

Triage Anhand der Symptome und des resultierenden diagnostischen Ductus bis zur primären Therapie kann das Geschehen bereits beim Erstkontakt mit dem Patienten eingeteilt werden in

- subakut,

- akut und

- perakut.

Daraus folgt eine Triage.
Perakutes Abdomen Eine unmittelbare vitale Bedrohung des Patienten (z.B. rupturiertes Aortenaneurysma) lässt keine Zeit für eine differenzialdiagnostische Abklärung.

- Nach einer nur minimalen Basisdiagnostik steht die operative oder interventionelle Versorgung im Vordergrund.

Dabei muss der Arzt ggf. eine gewisse „diagnostische Lücke“ in Kauf nehmen und die notwendige unmittelbare Erstversorgung veranlassen. Falsche oder verzögerte Entscheidungen können hier zum Tod führen.

Akutes Abdomen Beim akuten Abdomen im engeren Sinne handelt es sich um eine Gewebeverletzung - häufig begleitet von einer sympathiko-adrenergen Antwort. Anders als beim perakuten Abdomen ist hier noch eine basale, weitestgehend differenzierte Diagnostik möglich, z.B.

- radiologisch (CT etc.) oder

- analytisch (Urin, Labor).

Priorität hat dabei nicht die definitive Diagnosefindung, sondern die Bestimmung der erforderlichen Therapie (konservativ vs. chirurgisch vs. interventionell). Doch auch hier entscheidet der Faktor Zeit: Die Letalität des Patienten wird wesentlich bestimmt durch die zeitliche Verzögerung vom Einsetzen der ersten Symptome und, damit verbunden, einem möglichen Multiorganversagen [1].

Subakutes Abdomen Ein subakutes Abdomen ist oftmals verbunden mit

- dem Verlust der sympathischen Akutreaktion,

- aber erhöhter vegetativer Irritabilität mit kontinuierlichem, schubweisem oder chronischrezidivierendem Verlauf.

Das akute Abdomen ist eine vorläufige Bezeichnung für eine noch unklare abdominelle Erkrankung. In der Erstversorgung unterscheidet man

- subakutes,

- akutes oder

- perakutes Geschehen. 


\section{Schock}

Erstversorgung Das akute Abdomen ist häufig mit einem Schock verbunden. Falls noch nicht durch den einweisenden Notarzt erfolgt, ist Folgendes erforderlich:

- engmaschige Kreislaufkontrolle

- rasches Legen eines dicklumigen peripheren i.v. Zugangs mit Volumensubstitution

Die derzeitigen Sepsis-Leitlinien empfehlen für die Behandlung des septischen Schocks sowohl

- kristalloide als auch

- kolloidale Infusionslösungen.

Zudem können

- die Gabe von Katecholaminen und

- eine $\mathrm{O}_{2}$-Substitution

indiziert sein, seltener evtl. eine

- sofortige Intubation mit Beatmung.

Essenziell ist die

- frühzeitige Gabe von i.v. Breitspektrum-Antibiotika, möglichst innerhalb der ersten Stunde.

Eine rasch einsetzende Schocktherapie schafft Zeit für die spätere gezielte Diagnostik und vor allem die Sanierung des septischen Fokus.

Weitere Maßnahmen Parallel kann man erste diagnostische und therapeutische Maßnahmen sinnvoll koordinieren, so z. B. zwischen

- Schockraum,

- CT,

- Endoskopie,

- interventioneller Radiologie und

$>\mathrm{OP}$.

Wie viel Zeit hierfür zur Verfügung steht, richtet sich wiederum nach der Schwere des Krankheitsbildes: In der Regel kann es bereits zu diesem Zeitpunkt einem der 3 oben genannten Schweregrade (subakutes, akutes und perakutes Abdomen) zugeordnet werden.

Cave Der septische Schock ist zudem durch eine Alteration der koagulatorischen/antikoagulatorischen Balance charakterisiert: Es liegt ein eher pro-koagulatorischer Phänotyp vor [2], was bei den folgenden interventionellen oder chirurgischen Therapien berücksichtigt werden muss.

\section{Schmerztherapie}

\section{$\nabla$}

Analgetika Die aktuelle Literatur hat klar herausgestellt, dass der primäre Einsatz von Analgetika - nach Erhebung und Dokumentation des Abdominalbefundes - effizient ist. Analgetika beeinflussen weder die weiteren klinischen Untersuchungen oder das diagnostische Prozedere, noch verzögern sie den chirurgischen Eingriff [3].
Therapieren Sie den akuten abdominellen

Schmerz frühzeitig mit i.v. Analgetika.

\section{Interdisziplinarität \\ $\boldsymbol{\nabla}$}

Beschleunigte Diagnostik Die effiziente und zeitgerechte Erstversorgung des akuten Abdomens erfordert die Zusammenarbeit von

- Notfallmedizin,

- Anästhesie,

- Chirurgie,

- Innerer Medizin,

- Radiologie,

- Urologie,

- Gynäkologie,

- Neurologie und

- Orthopädie.

Ziel ist dabei, die Diagnostik zu beschleunigen und Risikopatienten schneller zu identifizieren.

Schockraum Beim akuten Abdomen ist die Koordination des Schockraum-Managements zwischen den einzelnen Abteilungen nicht nur sinnvoll, sondern obligat. Idealerweise beurteilen die jeweils erforderlichen Fachrichtungen die Situation bereits bei Einlieferung des Patienten im Schockraum. So können die Ärzte ein gemeinsames therapeutisches Konzept eröffnen.

Die effiziente und zeitgerechte Erstversorgung erfordert eine interdisziplinäre Zusammenarbeit der verschiedenen Fachabteilungen.

Aufgrund der Zeitnot müssen die Ärzte oft simultan operativ oder operativ-interventionell vorgehen, z. B.

- bei der gemeinsamen Versorgung des akuten Trauma-Patienten durch Viszeral-, Thorax-, Unfall- und Neurochirurgie oder

- bei der interdisziplinären diagnostischen Laparoskopie durch Gynäkologie und Viszeralchirurgie (z.B. bei akuter Unterbauchperitonitis der Frau).

Benefit vs. Risiko Interdisziplinarität bedeutet auch, Benefit und Risikopotenzierung für den Patienten abzuwägen. Gerade bei Trauma-Patienten gilt als fundamentales Prinzip die abgestufte Primärversorgung („Damage Control“) [4]. Die vollständige Definitivversorgung („Early total Care“) ist dagegen eher nicht anzustreben: Sie ist oft komplikationsträchtiger (siehe S. 30).

Endoskopisch-radiologisch-interventionelle Behandlung Auch bei den häufigsten endoskopisch-radiologisch-interventionellen Behandlungen des akuten Abdomens ist ein interdisziplinäres Management angesagt. Dazu zählen z.B. 


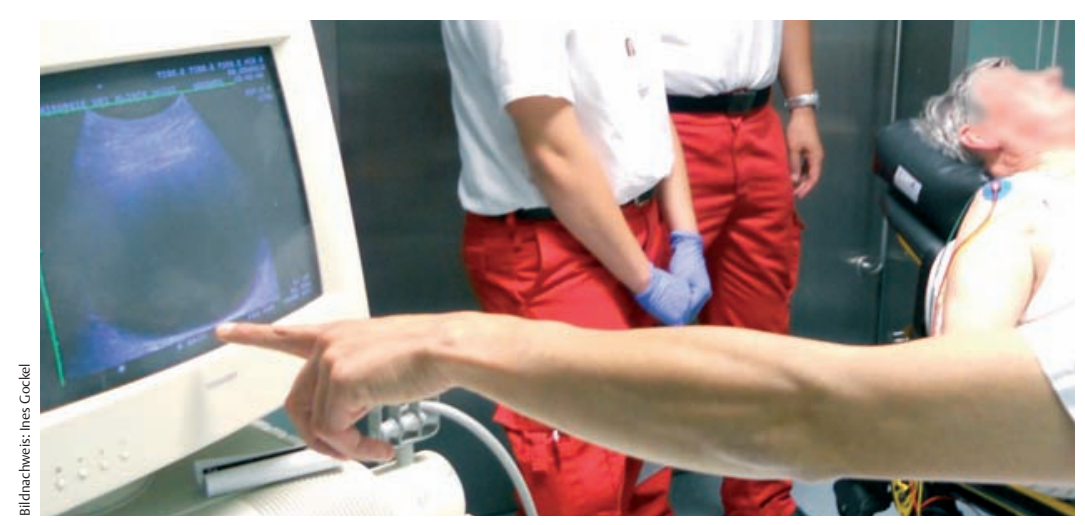

Abb. 1 Einweisungsdiagnose vom Notarzt: „gedeckt rupturiertes Aortenaneurysma“. Die Primärdiagnostik mittels Sonografie zeigt einen akuten Harnverhalt. Nach Anlage eines transurethralen Blasenkatheters entleeren sich spontan $2000 \mathrm{ml}$ Urin und der Patient ist beschwerdefrei - somit „pseudoakutes" Abdomen.

- spezielle Indikationen der akuten Mesenterialischämie,

- der akute Gallengangsverschluss,

- intraabdominelle Abszesse [5] und

- die endoskopische Primärversorgung akuter gastrointestinaler Blutungen.

Ein Chirurg sollte einbezogen werden und bei den entsprechenden Prozeduren bereit stehen, um
Tab. 1 Modifiziert nach [6]. sofort eine Operation veranlassen zu können, falls die interventionellen Maßnahmen nicht erfolgreich sind.

Beispiel: Obere gastrointestinale Blutung Bei einer akuten oberen gastrointestinalen Blutung ist eine Notfallgastroskopie indiziert. Gleichzeitig wird der Schockzustand durch Volumensubstitution therapiert. Wenn dann

- eine endoskopische Blutstillung nicht erfolgreich ist und

- der Patient mehr als 4-6 Blutkonserven pro $24 \mathrm{~h}$ zur Stabilisierung des Kreislaufs benötigt, so muss unverzüglich die Indikation zur Operation gestellt werden. Ziel ist die definitive, möglichst risikoarme Blutstillung im Sinne einer 4-Quadranten-Umstechung des blutenden Ulcus ventriculi - im Fall eines Ulcus duodeni mit Liga-

Nicht operationspflichtige Befunde bei
akuten abdominellen Schmerzen
- akute Pankreatitis
- Pseudoperitonitis diabetica
- Non-Ulcer-Dyspepsie
- Reizdarmsyndrom
- unkomplizierte Magen- und Darmulzera
- akute Gastroenteritis
- virale Hepatitis
- chronisch-entzündliche Darmerkrankung
ohne Komplikationen
- mesenteriale Lymphadenitis
- akute Pyelonephritis
- akute Porphyrie
- intestinale Pseudoobstruktion
- hämolytische Krisen
Salpingitis
kardiologische und pulmonologische
Erkrankungen
- Fornixruptur

tur der A. gastroduodenalis, die oftmals die Blutung an der Bulbushinterwand speist. In Einzelfällen kann die Magenresektion (Billroth I oder II, Roux-Y-Rekonstruktion, etc.) als das sichere Verfahren erforderlich sein. Zur gastrointestinalen Blutung siehe auch S. 30.

\section{Das „pseudoakute“ Abdomen}

$\nabla$

Konservative Therapie Obwohl dies der eigentlichen Definition des akuten Abdomens widerspricht, ist in speziellen Situationen eine konservative Behandlung indiziert. Dies betrifft insbesondere das „pseudoakute“ Abdomen, das nicht operationspflichtig ist.

Beispiel: Harnverhalt Die $\triangle$ Abb. 1 zeigt die Sonografie bei einem 84-jährigen Patienten, der vom Notarzt unter der Verdachtsdiagnose „gedeckt rupturiertes Aortenaneurysma“ in die Notaufnahme eingewiesen wurde. Bei Erstversorgung in der Klinik zeigte der Patient stabile und normwertige Vitalparameter. Akute Schmerzen bestanden im Unter- und Mittelbauch, die Dynamik bezeichnete er als langsam-progredient. - Die sofortige Ultraschall-Diagnostik des Abdomens zeigte eine massiv gefüllte Harnblase bei Harnverhalt.

Die abdominale Aorta sowie die parenchymatösen Organe stellten sich unauffällig dar, freie intraabdominelle oder retroperitoneale Flüssigkeit lag nicht vor. Dem Patienten wurde unverzüglich ein transurethraler Harnblasenkatheter angelegt, es entleerten sich $2000 \mathrm{ml}$ Urin. Die Beschwerden besserten sich sofort.

\section{Weitere Ursachen des „pseudoakuten“ Abdo-} mens Auch

- extraperitoneale Erkrankungen (Retroperitoneum, Bauchwand),

- extraabdominale Erkrankungen (Thorax, Skelett) und

- systemische Erkrankungen (Stoffwechselerkrankungen, hämatologische und neurologische Erkrankungen, Intoxikationen)

können ein „pseudoakutes“ Abdomen herbeiführen, das in seinen klinischen Ausprägungen primär nicht vom eigentlichen akuten Abdomen zu unterscheiden ist. Klassische Beispiele aus dem Gebiet der Inneren Medizin sind

- die basale Pneumonie,

- der Hinterwandinfarkt,

- der Diabetes mellitus,

- die Urämie und

- die akute Leukämie.

Sie sind häufige Ursachen der „Pseudoperitonitis“, die einer nicht-operativen Therapie bedürfen. Entsprechende Krankheitsbilder weiterer Disziplinen schließen den Kreis des „konservativen“ Therapiespektrums beim akuten Abdomen:

- Urologie (z.B. Zystitis) 
- Gynäkologie (z.B. Adnexitis, rupturierte Ovarialzyste)

- Neurologie (z.B. Radikulitis, Herpes zoster)

- Orthopädie (z.B. degenerative Arthritis, Osteomyelitis, Spondylodiszitis)

Weitere Beispiele sind in ๑ Tab. 1 aufgeführt.

Beim „pseudoakuten“ Abdomen ist eine konservative Therapie indiziert. Eine rasche Identifikation dieser nicht operationspflichtigen Befunde erspart dem Patienten die Negativ-Laparotomie.

\section{Erstversorgungsstrategien des akuten Abdomens}

\section{Infektion, Perforation, Peritonitis}

$\nabla$

Peritonitis Infolge einer

- Infektion (z.B. akute Appendizitis, Cholezystitis) oder

- Hohlorganperforation (Ulcus ventriculi bzw. duodeni, Sigmaperforation)

kommt es rasch zur lokalisierten und später generalisierten Peritonitis. Die gastrointestinale Perforation verursacht auch heute noch eine beträchtliche Mortalität und erfordert eine unverzügliche Notfalloperation [6].

Eindeutig ist der Befund im folgenden Beispielfall:

- plötzlich beginnende akute Oberbauchschmerzen („Vernichtungsschmerz“)

- bretthartes Abdomen

- Röntgenbild des Thorax im Stehen zeigt massiv freie Luft unter beiden Zwerchfellen ( $\bullet$ Abb. 2)

Bei der sofortigen Notfalloperation sah man ein frei perforiertes Ulcus ventriculi ad pylorum. Es wurde exzidiert und übernäht, gleichzeitig wurde das Abdomen gespült und eine Drainage eingelegt. Da im Exzidat Helicobacter pylori nachzuweisen war, wurde unmittelbar postoperativ eine Triple-Therapie zur Eradikation durchgeführt.
Diagnostische Laparoskopie Nicht immer ist die Diagnose „freie intraabdominelle Luft" so eindeutig. Häufig ist sie nur diskret in der Röntgenübersichtsaufnahme des Abdomens in Linksseitenlage zu erkennen. Auch die Abdomensonografie ist in diesem Fall limitiert. Hier kommt bei entsprechender Klinik der weiterführenden CT-Diagnostik oder auch der diagnostischen Laparoskopie eine besondere Bedeutung zu.

- Die diagnostische Laparoskopie hat in den letzten Jahren eine feste Position im Management des akuten Abdomens eingenommen.

Verglichen mit bildgebenden Verfahren oder wiederholten klinischen Untersuchungen erlaubt sie in den meisten Fällen eine definitive Diagnose $[7,8]$. Somit ergeben sich

- nicht nur diagnostische,

- sondern gleichzeitig therapeutische Optionen,

- und die Rate negativer Laparotomien kann gesenkt werden.

Appendizitis Bedingt durch die variable Lage der Appendix kann auch hier die diagnostische Laparoskopie hilfreich sein. Therapie der Wahl bei der akuten Appendizitis ist die minimalinvasive Appendektomie ( $\triangle$ Abb. 3 ).

Cholezystitis Die akute Cholezystitis wird frühelektiv (d.h. innerhalb von $72 \mathrm{~h}$ ) und überwiegend minimalinvasiv behandelt. Der Arzt sollte in der Anamnese den exakten Beginn der rechtsseitigen Oberbauchschmerzen erfassen. Ein primär offenes Vorgehen bei der akuten Cholezystitis ist nur selten indiziert, z. B.

- bei Patienten mit bekannten anatomischen Varianten des Gallengangs oder der Gefäßversorgung,

- nach mehrfachen Voroperationen oder

- wenn eine starke Blutungsneigung aus dem Leberbett zu erwarten ist (z. B. bei hämatologischen Patienten mit Thrombopenie oder sonstigen Gerinnungsstörungen).
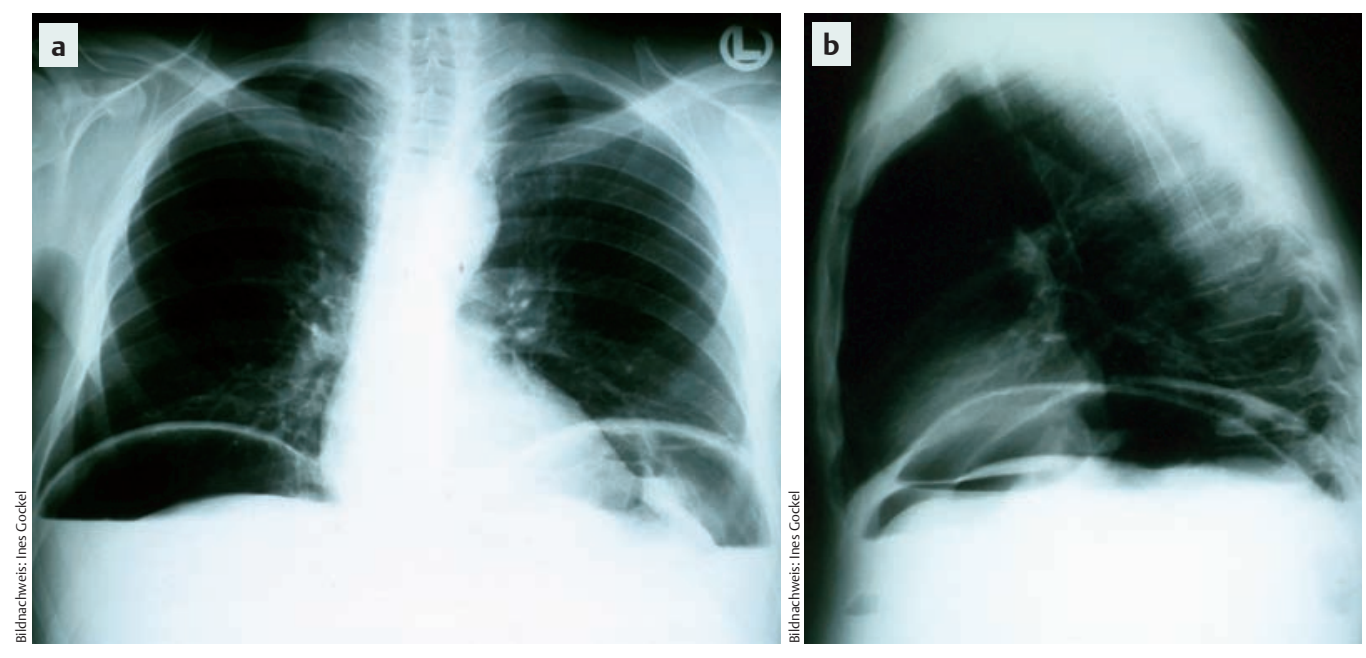

Abb. 2a,b Massiv freie Luft unter beiden Zwerchfellen bei Ulcus-ventriculi-Perforation. Links Frontalansicht (a.-p.), rechts Seitenansicht. 


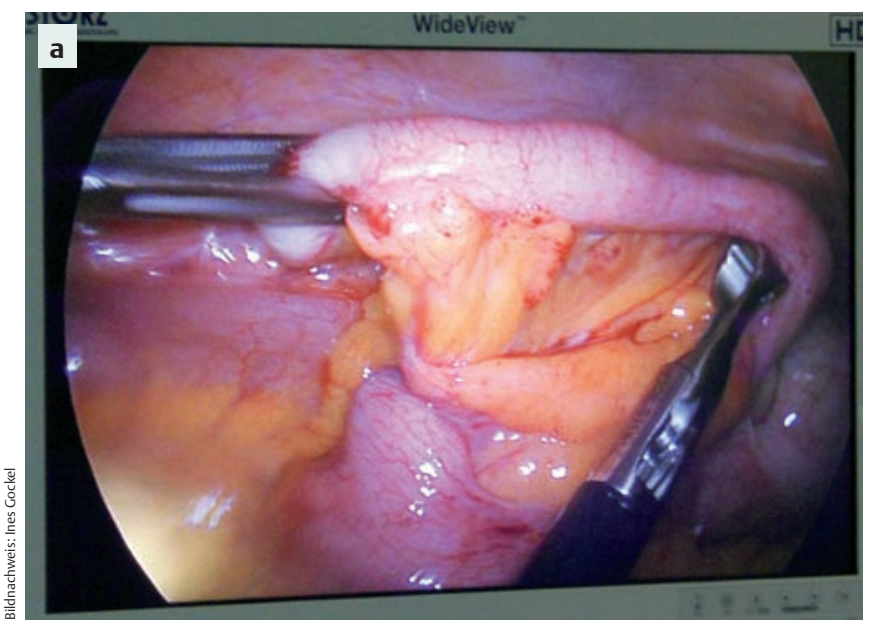

Abb. 3a,b Therapie der Wahl bei der akuten Appendizitis: Minimalinvasive Appendektomie.

Abb. 4 Im CT mit rektaler Füllung zeigt sich eine gedeckt perforierte Sigmadivertikulitis. Hier kann zunächst eine konservative Therapie indiziert sein, mit sekundärer laparoskopisch assistierter Sigmaresektion und primärer Anastomosierung im Intervall. nostik.

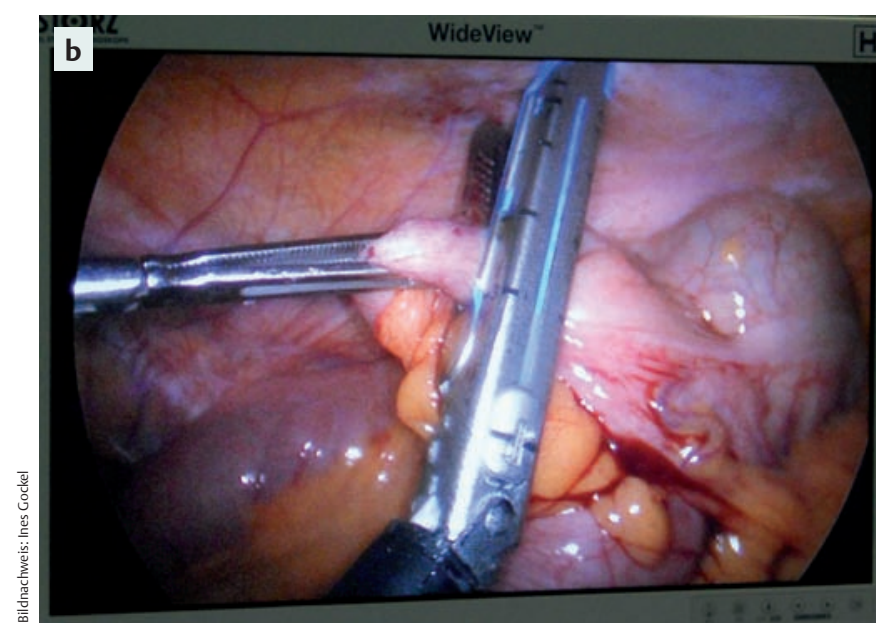

Sigmaperforation Wurde im CT mit rektaler Füllung die gedeckte Perforation einer Sigmadivertikulitis nachgewiesen (๑Abb.4), kann zunächst eine konservative Therapie mit Nahrungskarenz und i.v. Antibiose gerechtfertigt sein. Der Vorteil dieses Vorgehens: Man kann später eine laparoskopisch assistierte Sigmaresektion im symptomfreien Intervall durchführen und eine Stomaanlage vermeiden. Im Zweifelsfall ist aber auch hier eine diagnostische Laparoskopie indiziert, etwa bei klinisch deutlicher Peritonitis trotz fehlendem Kontrastmittelaustritt in der CT-Diag-

Bei Verdacht auf eine akute intraabdominelle Infektion oder Perforation kann in unklaren Situationen die diagnostische Laparoskopie indiziert sein.

Die freie Sigmadivertikelperforation ( $\bullet$ Abb. 5) mit eitriger oder kotiger Peritonitis hingegen erfordert die sofortige Notfalloperation. In den meisten Fällen erfolgt dies konventionell, es wird eine primäre Anastomose angelegt oder eine Hartmann-Situation geschaffen [9].

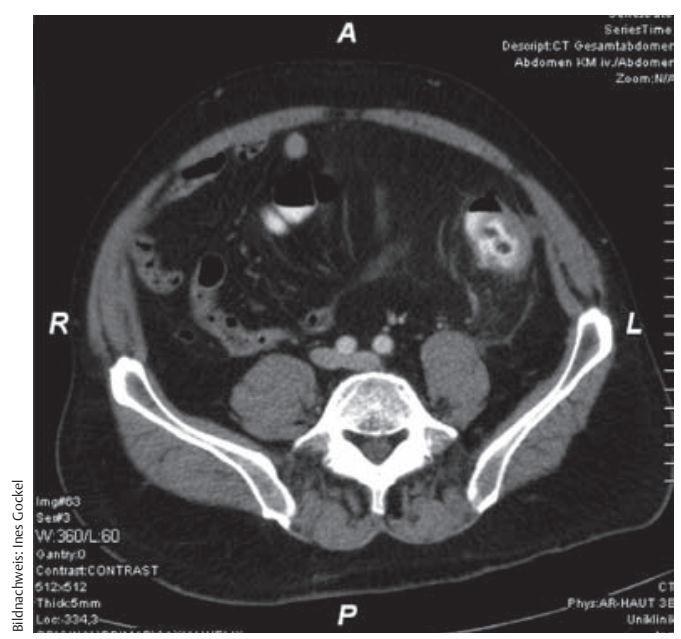

\section{Ileus: Bridenileus, Volvolus, Inkarzeration \\ $\nabla$}

Röntgendiagnostik bei lleus Nach Burkill et al. [10] ist die radiologische Bildgebung nur beim ausgeprägten und fortgeschrittenen Ileus hoch sensitiv (94\%) und spezifisch (96\%). Hier müssen also weitere Kriterien zur Entscheidungsfindung beitragen - und das schnell, denn eine verzögerte Operation bei Ileus kann zu einer Strangulation des Darms mit konsekutiver Nekrose führen, abgesehen von der prolongierten Gefahr der Aspiration. Hilfreich sind die Anamnese und die Inspektion des Abdomens hinsichtlich Narben bzw. Voroperationen:

- Gab es Voroperationen, liegt wahrscheinlich ein Bridenileus des Dünndarms vor.

- Bei der Differenzialdiagnostik der Obstruktion des Kolons handelt es sich oftmals um ein stenosierendes Karzinom.

Gastrographin umstritten Bei unklarer Situation und Spiegelbildung in der Röntgen-Leeraufnahme ist die Durchführung einer Gastrographinpassage umstritten. Vorteile sind möglicherweise - eine Lokalisation der Obstruktion und - eine Differenzierung des Ileus-Grades. Unbestritten ist auch der therapeutische Effekt von wasserlöslichem Kontrastmittel beim inkompletten Dünndarmileus [11]. Nachteile sind jedoch

- die Gefahr des Erbrechens mit Aspiration,

- die lange Dauer der Untersuchung und

- häufig eine fehlende Klärung der Ursache.

Zeigt sich $6 \mathrm{~h}$ nach Gastrographingabe noch kein Progress des Kontrastmittels im oberen Gastrointestinaltrakt bei dilatierten Dünndarmschlingen, ist die Laparotomie - bzw. bei nicht vorhandenen Voroperationen die Laparoskopie - indiziert.

Volvolus Ein episodenartiger, intermittierender und heftiger Schmerz kennzeichnet

- einen Dünndarmvolvolus (bedingt z.B. durch eine innere Hernie mit intermittierender Dünndarm-Inkarzeration) oder 

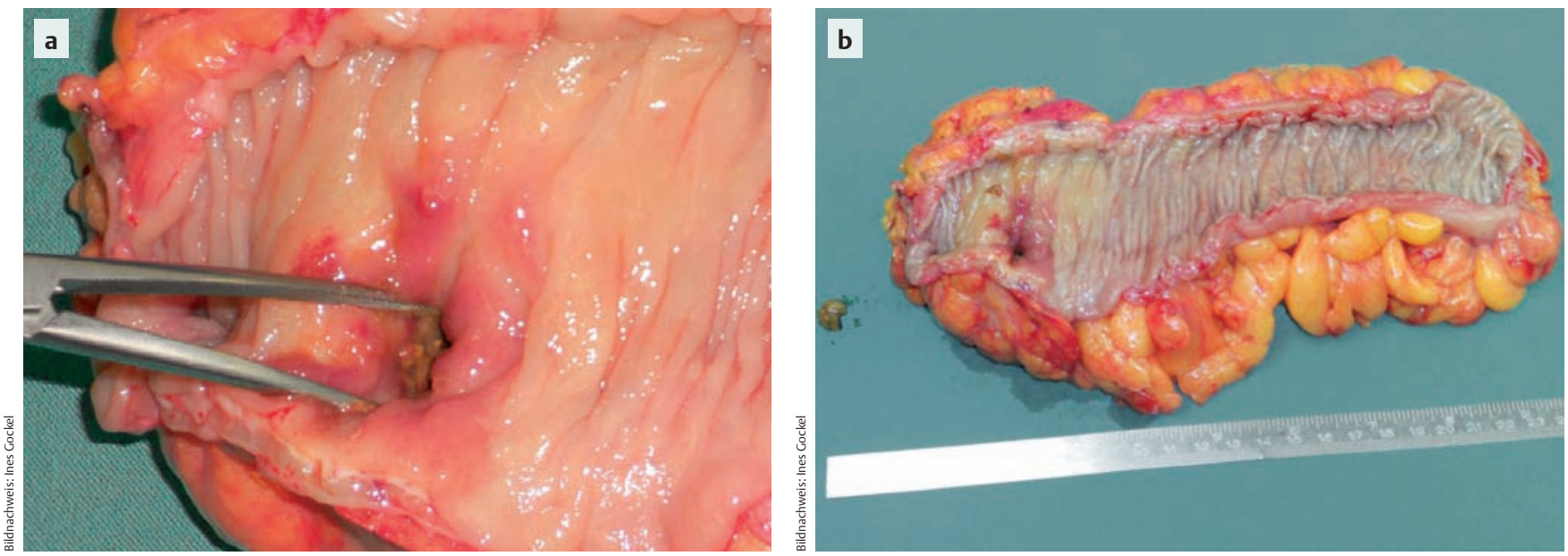

- einen Sigmavolvolus bei Sigma elongatum (o Abb. 6).

Erfolgt die Röntgendiagnostik im symptomfreien Intervall, bleibt sie möglicherweise negativ.

Inkarzeration Die Inkarzeration durch äußere Hernien ist einfach zu diagnostizieren und bedarf nach erfolgreicher Reposition nicht immer einer sofortigen operativen Therapie.

Cave En-bloc-Reposition von Bruchsack und Bruchpforte mit persistierender Inkarzeration des Bruchsackinhalts.

Jedoch sollte man die Hernie dann früh-elektiv operieren, um eine rezidivierende Einklemmung zu vermeiden. Seltener sind Inkarzerationen durch innere Hernien, wie z. B. beim Enterothorax (० Abb. 7, 8).

\section{Trauma}

Der klinische Verlauf des Polytraumas wird maßgeblich von der Behandlungsstrategie innerhalb der ersten $24 \mathrm{~h}$ nach dem Trauma beeinflusst [12].

Stumpfes Trauma Nach stumpfem Abdominaltrauma ist eine Laparotomie - ggf. in unklarer Situation eine diagnostische Laparoskopie - indiziert, sofern

- die Verletzung kontinuierlich blutet,

- Substitutionsbedarf durch eine intraabdominelle Hämorrhagie besteht (Leber- oder Milzruptur, Mesenterialgefäßeinriss) und

- der Patient hämodynamisch instabil ist.

Als Grenzwert gilt neben dem klinischen Status (z.B. Schweregrad der Leberruptur nach Moore: Grad III) das Vorliegen entsprechender radiologischer Hinweise für eine ausgedehnte Organverletzung [12].
Ziel der operativen Maßnahmen ist die Kontrolle einer vital-bedrohlichen intraabdominellen Blutung und somit die Wiederherstellung und Sicherung des Kreislaufs.

Cave Kontrollieren Sie intraoperativ bei einem stumpfen Trauma mit Leber- oder Milzruptur immer auch alle anderen Organe und Strukturen! Oft ist ein Zweitbefund vorhanden, z. B. eine Zwerchfellruptur oder ein Mesenterialeinriss.

Polytrauma Bei polytraumatisierten Patienten mit

- schweren intraabdominellen Blutungen,

- konsekutiver Gerinnungsstörung und

- prolongiertem Schock

sollte die initiale chirurgische Therapie ausschließlich der Blutstillung dienen (z.B. intraabdominelles Packing der Leber mit Bauchtüchern). Die definitive Versorgung bzw. Rekonstruktion kann später erfolgen, nach einer Phase der Stabilisierung der Vitalparameter und der Gerinnung („Damage Control“) [13,14]. Patienten mit früher vollständiger Definitivversorgung („Early total Care“) leiden dagegen oft unter

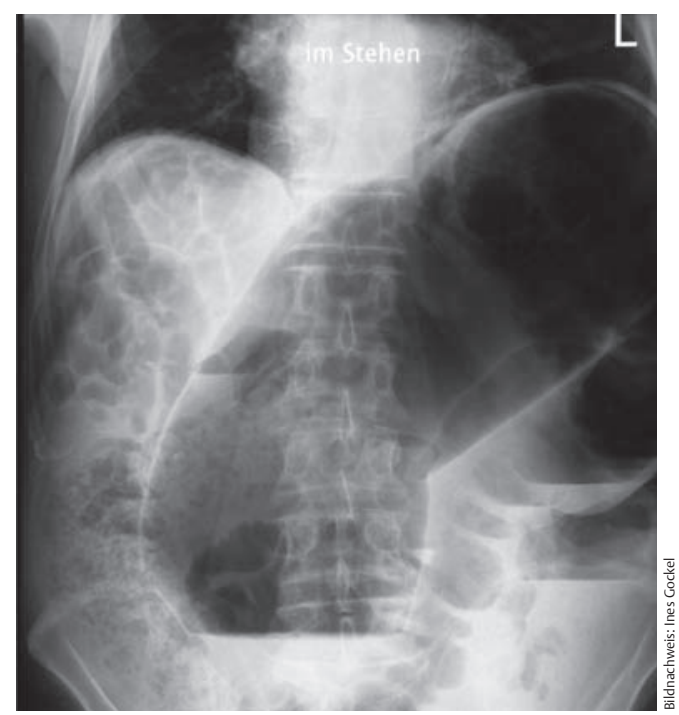

Abb. 5a, b Intraoperativer Befund einer freien Sigmadivertikelperforation.

Abb. 6 Sigmavolvolus bei Sigma elongatum. In der Röntgenübersichtsaufnahme des Abdomens im Stehen zeigt sich ein deutlicher Dickdarmileus bei Sigmavolvolus. 
Bildnachweis: Ines Gockel

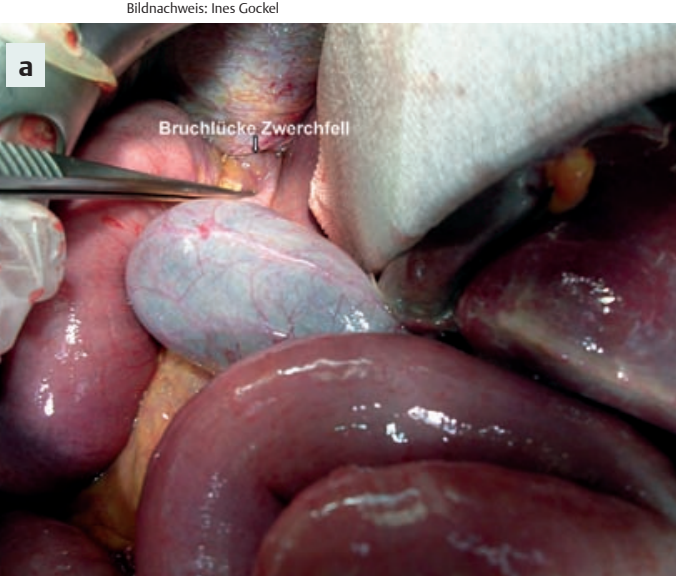

Bildnachweis: Ines Gockel

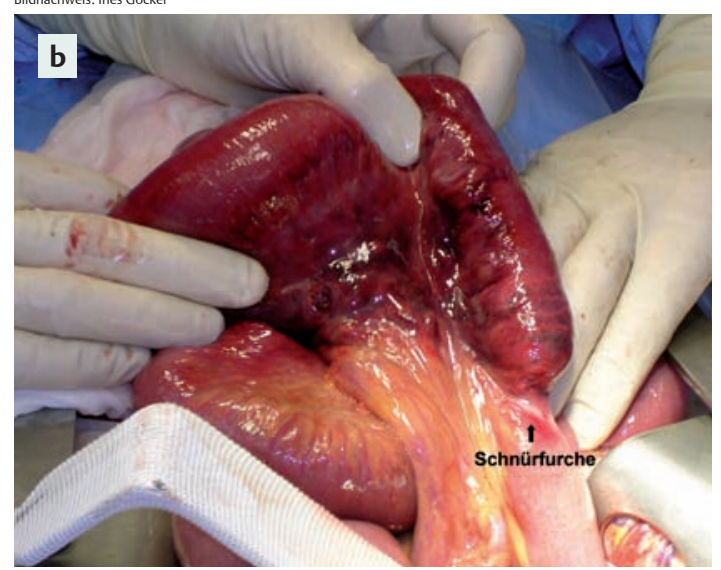

Bildnachweis: Ines Gockel

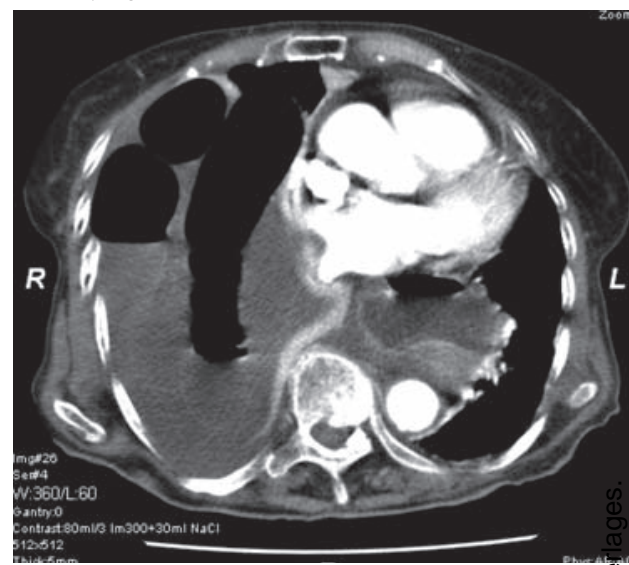

Abb. 7a,b Beginnende Gangrän des Dünndarms bei Enterothorax und Zwerchfellhernie.

Abb. 8, rechts Innere Hernie mit Inkarzeration von Dünndarm bei Enterothorax und Zwerchfellhernie. Präoperativer CT-Befund.
- Gerinnungsstörungen,

- inflammatorischen Reaktionen und

- Hypothermie.

Dadurch können gravierende Komplikationen entstehen: vom Multiorganversagen über das "Acute Respiratory Distress Syndrome“ (ARDS) bis zum Tod.

Beim akuten Polytrauma ist die „Damage Control“ risikoärmer als die „Early total Care“.

\section{Vaskuläre Ursachen}

Hohe Mortalität Die abdominellen vaskulären Notfälle sind selten, jedoch mit einer hohen Letalität verbunden - trotz verbessertem Verständnis der Pathophysiologie dieser Erkrankungen. Die wichtigsten Faktoren für die hohe Mortalität sind Verzögerungen in Diagnostik und Erstversorgung [15].

Mesenteriale Ischämie Die $\bullet$ Abb. 9 zeigt den operativen Situs einer 42-jährigen Patientin mit mesenterialer Ischämie auf dem Boden einer Thrombophilie. Die zuvor angefertigte Röntgenuntersuchung des Abdomens ergab eine ausgeprägte Pneumatosis intestinalis ( Abb. 10). Unter den Ursachen der akuten mesenterialen Ischämie findet sich
- am häufigsten der arterielle Verschluss,

- gefolgt von der nicht-okklusiven und venösen Genese

- sowie der Aortendissektion.

Ein Angio-CT kann die mesenteriale Ischämie zwar mit hoher Sicherheit ausschließen (negativer Vorhersagewert 95\%), aber auch hier ist bei klinischem Verdacht immer die diagnostische Laparoskopie, ggf. Laparotomie, indiziert.

Bei thrombembolisch bedingter mesenterialer Ischämie nimmt die kathetergestützte thrombolytische Therapie als unterstützendes Verfahren an Bedeutung zu [16].

Die akute mesenteriale Ischämie ist durch das charakteristische symptomfreie Intervall schwierig zu diagnostizieren. Aufgrund des kurzen Zeitfensters und der hohen Mortalität ist bei klinischem Verdacht immer die Notfalloperation indiziert.

\section{Gastrointestinale Blutung}

$\nabla$

Endoskopische Blutstillung Bei der oberen (ca. 80-90\% aller Fälle) und unteren gastrointestinalen Blutung ist immer zunächst als 1 . Schritt eine endoskopische Blutstillung indiziert (z. B. Clipsetzung, Suprarenin-Unterspritzung). Auf diese Situation wurde bereits oben unter „Interdisziplinarität" eingegangen.
Abb. 9, links Operativer Situs einer 42-jährigen Patientin: Kolonischämie und Nekrose der distalen $50 \mathrm{~cm}$ des lleums, toxisches Megakolon auf dem Boden einer Thrombophilie.

Abb. 10, rechts Röntgenübersichtsaufnahme des Abdomens mit ausgeprägter Pneumatosis intestinalis.
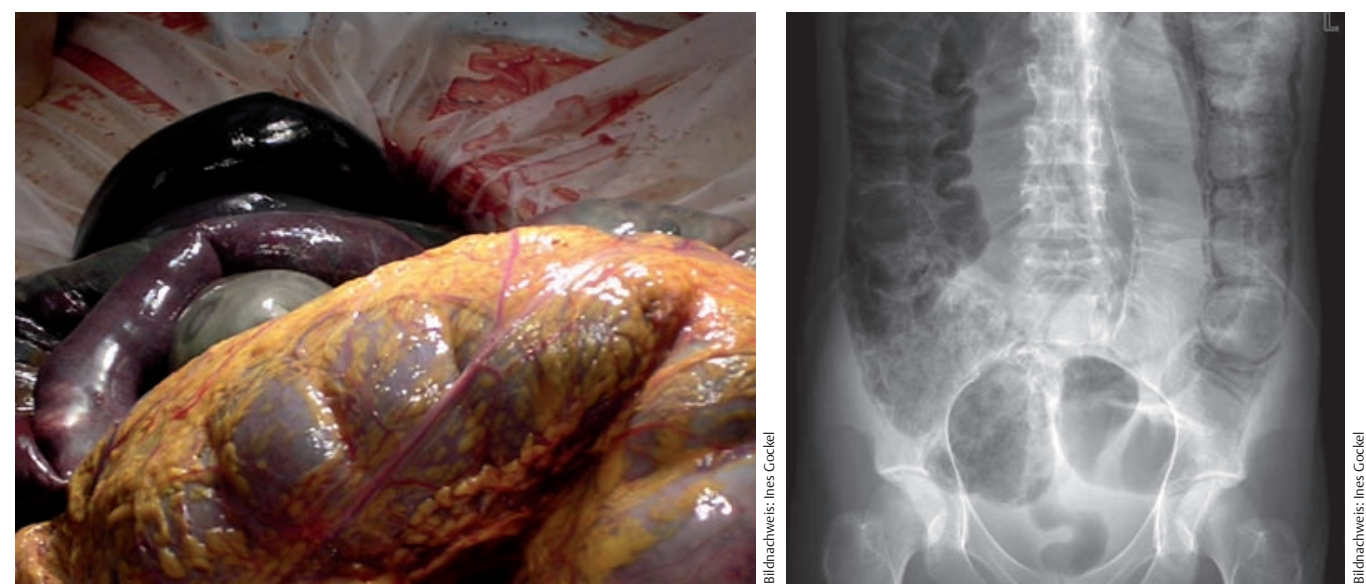
Embolisation Als Alternative zur Endoskopie kommt vor einer chirurgischen Therapie auch die radiologisch-interventionelle Embolisation zum Einsatz. Sie bietet sich an bei

- arteriellen Blutungen aus dem Gastrointestinaltrakt, aus der Leber, aus dem Darm (z.B. infolge eines Aneurysmas oder einer vaskulären Malformation) sowie bei

- blutenden intestinalen Anastomosen [16].

Auch hier ist immer eine engmaschige interdisziplinäre Absprache erforderlich, um zeitgerecht die Indikation zur chirurgischen Versorgung zu stellen, falls die interventionellen Maßnahmen erfolglos bleiben. Liegt ein massiver hämorrhagischer Schock vor, so hat die operative Notfallversorgung höchste Priorität.

Die akute gastrointestinale Blutung wird interdisziplinär versorgt. Man richtet sich dabei nach

- Ausmaß des Blutverlustes,

- Zahl der substituierten Konserven,

- Lebensalter des Patienten,

- Begleiterkrankungen sowie

- Schweregrad und Dauer des hämorrhagischen Schocks.

Interessenkonflikt Die Autoren erklären, dass keine wirtschaftlichen oder persönlichen Verbindungen bestehen.

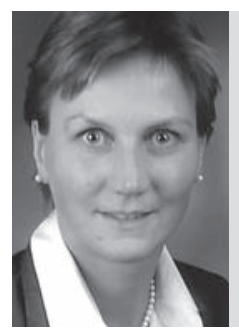

PD Dr. med. Ines Gockel ist Oberärztin an der Klinik und Poliklinik für Allgemein- und Abdominalchirurgie der Johannes-Gutenberg-Universität Mainz. E-Mail:

ines.gockel@unimedizin-mainz.de

Martin Schröder ist Assistenzarzt an der Klinik und Poliklinik für Allgemein- und Abdominalchirurgie der Johannes-Gutenberg-Universität Mainz.

E-Mail: schroeder@ach.klinik.uni-mainz.de

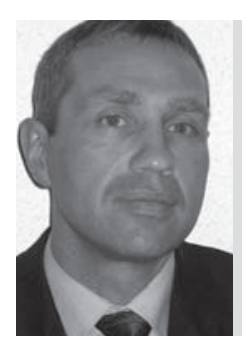

Univ.-Prof. Dr. med. Hauke Lang ist Direktor der Klinik und Poliklinik für Allgemein- und Abdominalchirurgie der Johannes-Gutenberg-Universität Mainz. E-Mail: lang@ach.klinik.uni-mainz.de

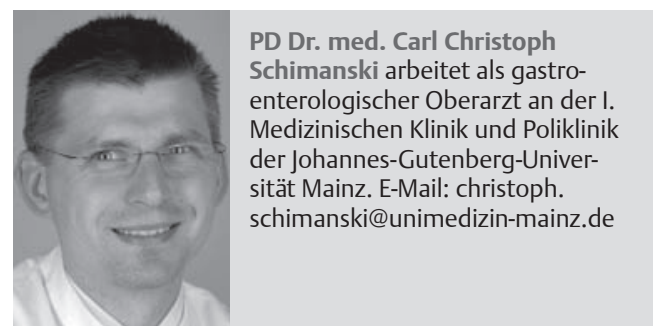

Fazit Beim akuten Abdomen muss der Arzt potenziell lebensbedrohliche Situationen schnell erkennen und ggf. auf differenzierte Diagnostik verzichten. Die interdisziplinäre Zusammenarbeit ist für eine zeitnahe Diagnose und Therapie essenziell. Die diagnostische Laparoskopie hat in dieser Situation eine herausragende Bedeutung.

\section{Kernaussagen}

- Richten Sie Ihr Vorgehen nach Ihrer Einschätzung beim Primärkontakt mit dem Patienten und der daraus folgenden Einteilung in ein

$\triangleright$ akutes,

$\triangleright$ perakutes oder

$\triangleright$ subakutes Abdomen.

- Im Falle

$\triangleright$ einer Infektion,

$\triangleright$ einer Hohlorganperforation,

$\triangleright$ einer lokalen oder einer generalisierten Peritonitis

liegt der Schwerpunkt auf der raschen Sanierung des Fokus. Um ihn zu identifizieren, ist ggf. eine diagnostische Laparoskopie notwendig.

- Liegt ein lleus vor, gilt das primäre Augenmerk der Beseitigung des mechanischen Hindernisses. Auf den Bridenileus können abdominelle Voroperationen hinweisen.

- Beim polytraumatisierten Patienten ist oftmals die „Damage Control“ der frühen vollständigen Definitivversorgung („Early total Care“) vorzuziehen.

- Bei begründetem Verdacht auf eine mesenteriale Ischämie ist die unverzügliche Notfalloperation indiziert.

- Bei einer gastrointestinalen Blutung wird der Patient zunächst stabilisiert und die Blutung endoskopisch versorgt. Ist sie endoskopisch nicht kontrollierbar, wird sie chirurgisch behandelt.

\section{Literatur}

1 Torer N, Yorganci N, Elker D, Sayek I. Prognostic factors of the mortality of postoperative intraabdominal infections. Infection 2010; 38: 255-260

2 Russel JA. The current management of septic shock. Minerva Med 2008; 99: 431-458

3 Schreyer N, D’Ambrogio A, Demartines N. Acute abdominal pain: what about analgesia. Rev Med Suisse 2007; 3: 1647-1650

4 Kushimoto S, Miyauchi M, Yokota H, Kawai M. Damage control surgery and open abdominal approach: Recent advances and our approach. J Nippon Med Sch 2009; 76: 280-290

5 Trumm C, Hoffmann RT, Reiser MF. Radiological interventional procedures for the acute abdomen. Radiologe 2010; 50: 262-271

6 Langell JT, Mulvihill SJ. Gastrointestinal perforation and the acute abdomen. Med Clin North Am 2008; 92: 599-625

7 Keller R, Kleemann M, Hildebrand P, Roblick UJ, Bruch HP. Diagnostic laparoscopy in acute abdomen. Chirurg 2006; 77: 981-985

8 Stefanidis D, Richardson WS, Chang L, Earle DB, Fanelli RD. The role of diagnostic laparoscopy for acute abdominal conditions: an evidence-based review. Surg Endosc 2009; 23: 16-23

9 Germer C, Groß V. Diverticulitis: When to treat medically, when surgically? Dtsch Arztebl 2007; 104: A-3486

10 Burkill G, Bell J, Healy J. Small bowel obstruction: the role of computed tomography in its diagnosis and management with reference to other imaging modalities. Eur Radiol 2001; 11: 1405-1422

11 Graeb C, Reiser M, Jauch KW, Graser A. Acute abdomen. Clinical background and demands on imaging. Radiologe 2010; 50: 209-213
Beitrag online zu finden unter http://dx.doi.

org/10.1055/

$\underline{\mathrm{s}-0031-1272351}$

\section{Literatur online}

Das vollständige Literaturverzeichnis zu diesem Beitrag finden Sie im Internet:

Abonnenten und Nichtabonnenten können unter „www.thieme-connect.de/ejournals" die Seite der Lege artis aufrufen und beim jeweiligen Artikel auf „Ergänzendes Material" klicken - hier ist die Literatur für alle frei zugänglich.

Abonnenten können alternativ über ihren persönlichen Zugang an das Literaturverzeichnis gelangen. Wie das funktioniert, lesen Sie unter: http:// www.thieme-connect.de/ ejournals/ help\#SoRegistrieren 